\title{
Retraction: DNA-guided genome editing using the Natronobacterium gregoryi Argonaute
}

Feng Gao, Xiao Z Shen, Feng Jiang, Yongqiang Wu \& Chunyu Han

Nat. Biotechnol. 34, 768-773 (2016); published online 2 May 2016; addendum published after print 28 November 2016 ; retracted 2 August 2017; doi:10.1038/nbt.3547

We are retracting our study because of the continued inability of the research community to replicate the key results in Figure 4, using the protocols provided in our paper. In this figure we report that the Natronobacterium gregoryi Argonaute can efficiently create double-strand breaks and edit the genome of human cells using 5' phosphorylated single-stranded DNA as a guide. Despite the efforts of many laboratories (Protein Cell 7, 913-915, 2016; Nat. Biotechnol. 35, 17-18, 2017; Cell Res. 26, 1349-1352, 2016; PLOS One 12, e0177444, 2017), an independent replication of these results has not been reported. We are therefore retracting our initial report at this time to maintain the integrity of the scientific record. We nevertheless continue to investigate the reasons for this lack of reproducibility with the aim of providing an optimized protocol.

\section{Erratum: Nature Biotechnologys academic spinouts of 2016}

Aaron Bouchie, Laura DeFrancesco, Cormac Sheridan \& Sarah Webb

Nat. Biotechnol. 35, 322-333 (2017); published online 11 April 2017; corrected after print 25 May 2017

In the version of this article initially published, on p.326, column 3, Shawn Marcell was described as "an economist turned pathologist," rather than "an economist and entrepreneur." Four lines below, his name was misspelled as "Marcel." On p.329, the thumbnail caption named Kuball CEO; he is CSO, as it says in the text. On p.330, column 3, David Pompliano was incorrectly said to be "a partner at Apple Tree Partners (New York)." On p.331, column 1, Exicure CEO Giljohann's first name was given as John in the caption; it is David. In column 2, paragraph 1 (and also in column 3, paragraph 3), AuraSense should have been AuraSense Therapeutics; paragraph 2, line 1, "AuraSense described" should have been "the Mirkin group described"; paragraph 4, line 1, "Exicure has published" should have been "The Mirkin group has published"; last paragraph, "upfront payment and equity investment" should have been "upfront payment and potential equity investment." In column 3, paragraph 1, "ectopic dermatitis" should have been "atopic dermatitis"; in paragraph 2, the last half of the last sentence, "to enter a phase 1 trial in combination with a checkpoint inhibitor to treat solid tumors in the first half of 2017" should have read "to enter a phase 1 safety study in healthy volunteers in the first half of 2017, and is expected to enter a trial in combination with a checkpoint inhibitor to treat solid tumors in the first half of 2018 ." The errors have been corrected in the HTML and PDF versions of the article.

\section{Erratum: Making individualized drugs a reality}

\author{
Huub Schellekens, Mohammed Aldosari, Herre Talsma \& Enrico Mastrobattista \\ Nat. Biotechnol. 35, 507-513 (2017); published online 7 June 2017; corrected after print 5 June 2017
}

In the version of this article initially published, the line "Editor's note: This article has been peer-reviewed." was omitted. The error has been corrected in the HTML and PDF versions of the article.

\section{Erratum: Genome-wide target specificities of CRISPR RNA-guided programmable deaminases}

\author{
Daesik Kim, Kayeong Lim, Sang-Tae Kim, Sun-heui Yoon, Kyoungmi Kim, Seuk-Min Ryu \& Jin-Soo Kim \\ Nat. Biotechnol. 35, 475-480 (2017); published online 10 April 2017; corrected after print 6 July 2017
}

In the version of this article initially published, in the HTML only, Daesik Kim should have been the second corresponding author rather than Seuk-Min Ryu. In Figure 4b, in all versions, the bar graphs were misaligned with the specificity ratios, so that the first row of bar graphs were above the specificity ratios, rather than aligned with 3.5, 1.0, etc. The errors have been corrected in the HTML and PDF versions of the article. 